ISSN 2079-9292

www.mdpi.com/journal/electronics

Article

\title{
Investigation of a Switchable Textile Communication System on the Human Body
}

\author{
Qiang Bai ${ }^{1}$, Srijittra Swaisaenyakorn ${ }^{2}$, Hyung-Joo Lee ${ }^{1}$, Kenneth L. Ford ${ }^{1}$, \\ John C. Batchelor ${ }^{2}$ and Richard J. Langley ${ }^{1, *}$
}

1 Department of Electrical and Electronic Engineering, University of Sheffield, Mappin Street, Sheffield S1 3JD, UK; E-Mails: q.bai@sheffield.ac.uk (Q.B.); melonicus@hotmail.com (H.-J.L.); 1.ford@sheffield.ac.uk (K.L.F.)

2 School of Engineering and Digital Arts, University of Kent, Canterbury, Kent CT2 7NT, UK; E-Mails: ss638@kent.ac.uk (S.S.); j.c.batchelor@kent.ac.uk (J.C.B.)

* Author to whom correspondence should be addressed; E-Mail: r.j.langley@sheffield.ac.uk; Tel.: +44-114-222-5583.

Received: 13 February 2014; in revised form: 4 June 2014 / Accepted: 14 July 2014 / Published: 18 August 2014

\begin{abstract}
In this paper, a switchable textile communication system working at $2.45 \mathrm{GHz}$ ISM band is presented and studied for different locations within a realistic on-body environment. A 3D laser scanner is used to generate a numerical phantom of the measured subject to improve the accuracy of the simulations which are carried out for different body postures. For the off-body communications, the system is acting as an aperture coupled microstrip patch antenna with a boresight gain of $1.48 \mathrm{dBi}$. On-body communication is achieved by using a textile stripline, which gives approximately $5 \mathrm{~dB}$ transmission loss over $600 \mathrm{~mm}$ distance. The system is switched between on and off-body modes by PIN diodes. Common issues, such as shape distortion and body detuning effects which the textile antenna may experience in realistic use are fully discussed. Robust antenna performance is noted in the on-body tests, and an additional $3 \mathrm{~dB}$ transmission coefficient deduction was noticed in the most severe shape distortion case.
\end{abstract}

Keywords: switchable body-centric communication; on-body communication; off-body communication; textile strip patch antenna; textile stripline 


\section{Introduction}

Recently, the textile antenna has attracted much attention as wearable electronics and smart clothes are becoming a popular topic in industry and academia. The idea of wearing an antenna on body can free the antenna system from the miniaturized wearable devices, and consequently enhance the wireless communication performance by fully utilizing the human body surface. Using new flexible materials, such as conductive textiles, can significantly reduce the antenna weight and seamlessly integrate the wearable antenna into clothes [1,2]. The antenna made of flexible materials needs to be optimized to remain satisfied performance under shape distortion conditions. In [3-5], wearable antennas were tested and discussed under bending and crumpling conditions. A statistical technique was also introduced to analyze the uncertainty of textile antenna resonance frequency after bending [6]. In realistic usage, textile antennas may need to be waterproofed when exposed to adverse environmental conditions as moisture can significantly affect the antenna performance $[7,8]$.

Body area networks involve two wireless communication scenarios. These are on-body communication, which refers to communication between nodes located in different parts of the human body, and off-body communication where signals are transmitted between the on-body node and the nodes far away from body such as wireless routers. The wearable antennas therefore need to be characterized for on and off body applications [1]. The antennas designed for off-body mode are for communications between the node mounted on body and the wireless router or base station at a distance from the wearer. Therefore, the antenna has to radiate mainly in the boresight direction. In [4,5,9-12], different types of wearable antennas were presented for off-body applications. Artificial magnetic conductors (AMCs) were used to enhance the antenna forward radiation and reduce the detuning effect from the human body. Some wearable antennas designed for on-body communication applications have been reported in [13-16], where the antennas need to have the maximum directivity along the body surface, not only to achieve a better on-body transmitting and receiving performance, but also to reduce the radio interference between nearby wearers. However, it has been observed that the Non Line of Sight (NLOS) on-body wireless link may experience a significant path loss. In [17], antennas were designed and compared for over body surface communication at $2.45 \mathrm{GHz}$, and the path loss is generally greater than $36 \mathrm{~dB}$ over a distance of $257 \mathrm{~mm}$ in NLOS cases. It will be a very attractive idea if these two communication scenarios can be integrated into one system, but only a small number of papers have studied such a switchable antenna or communication system which can support both on- and off-body communications [18-21].

In this paper, the switchable textile communication system briefly described in [20,22] operating in both on and off body modes at $2.45 \mathrm{GHz}$ is presented and investigated in a realistic body worn environment. The novelty of this work is that the performance is presented for different locations of the communication system on the body and for different body postures. Furthermore, a 3D laser scanner is used to generate a numerical phantom of the measured subject to improve the accuracy of the simulation, as described in Section 2. A textile stripline is employed in this communication system for on-body communications to provide the means to overcome hefty NLOS attenuation, which was also investigated under complex shape distortion conditions in this work. The system geometry is introduced in Section 3 and the performance of on- and off-body modes are investigated in Sections 4 and 5 , respectively. Conclusions are drawn in the final section. 


\section{Methodology}

The wearable communication system was designed and tested with a human body using both numerical and experimental methods. In order to increase the prediction accuracy of the antenna on-body performance, the numerical body phantom used in the simulation must have a minimum shape difference from the body of the measured test subject. The antenna in the simulation also needs to be conformal to the same shape and mounted in exactly the same on-body location as for the measurement. Therefore, three processes were involved in this study to evaluate the wearable antenna performance and to achieve accurate predictions.

\subsection{Creating a 3D-Body Model of the Test Subject}

A test subject participating in a measurement process was scanned by a 3D laser scanner. During the scanning process, the subject wore a skin-tight suit and remained still for approximately for $10 \mathrm{~s}$. A 3 column optical laser 3D body scanner with a data density of 7 points $/ \mathrm{cm}^{2}$ was used [23]. After the model was scanned, image reconstruction software from "Human Solution GmbH" was used to create a 3D surface of the model [24]. Areas with poor reflection or shadowing were subsequently fixed by post-processing process in the software (Figure 1). The 3D surface in ".obj" format was converted into a 3D solid model ".sat" format using "Mesh2Solid" software so it could be used later in the simulation process. This technique was shown in $[25,26]$ to demonstrate that a $3 \mathrm{D}$ scanned surface of a test subject could enhance the accuracy of the predicted transmission coefficients from EM simulator, CST Microwave Studio.

Figure 1. (a) The avatar reference person being $3 \mathrm{D}$ body scanned, and (b) the $3 \mathrm{D}$ scanned surface.

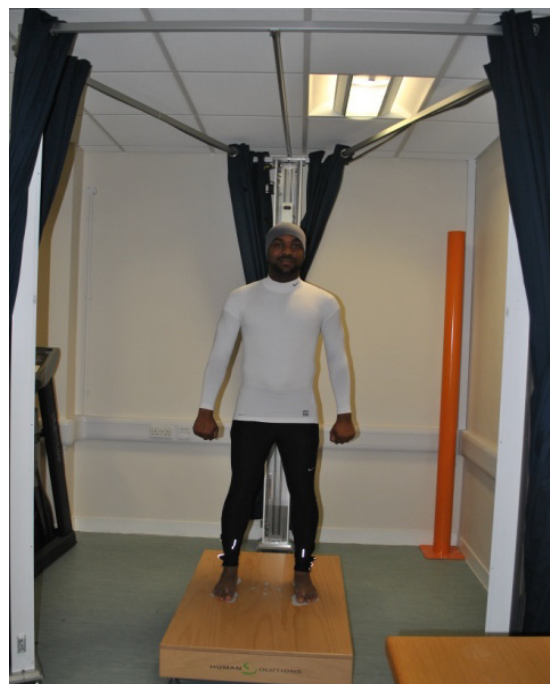

(a)

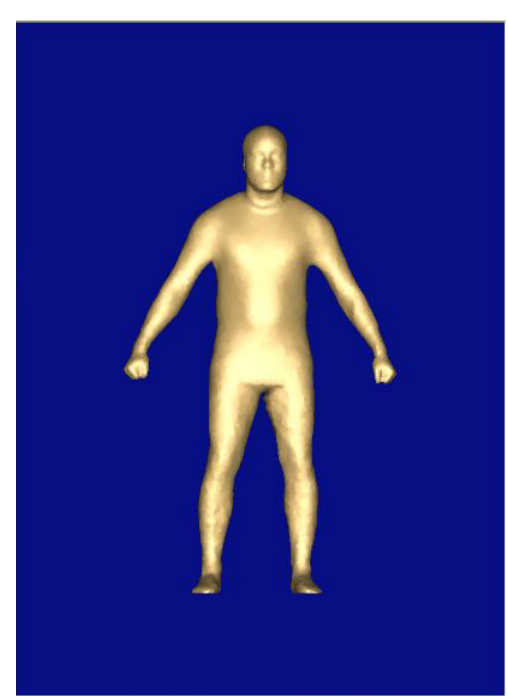

(b)

\subsection{Measuring the Wearable Antenna System Characteristics}

All measurements were taken using a textile stripline antenna, radiating at 2-3 GHz frequency band. The antenna was then connected to an FSH8 Rohde and Swartz network analyzer to measure the 
channel characteristics, $\left|S_{11}\right|$ and $\left|S_{21}\right|$. The measurements were taken first on a human torso and then on an equivalent sized polystyrene human torso model. This was done to isolate the free space channel between the two antennas from any effects caused by the body tissue electrical values and any changes caused in the radio channel characteristics when the antenna was in close proximity to the human body.

The antenna was attached around a volunteer's torso having one end placed around the stomach area. It was biased with a DC power supply to operate in an "off-body" mode (Figure 2). The torso area was $110.5 \mathrm{~cm}$ above the ground with a circumference of $102.5 \mathrm{~cm}$.

Figure 2. Stripline antenna bending around (a) a human's torso; (b) a polystyrene's torso; (c) diagonal bending cross the human chest. The white/grey balls in the figure indicate the motion capture markers, used to record the location and orientation of each part of the antenna during the measurement.

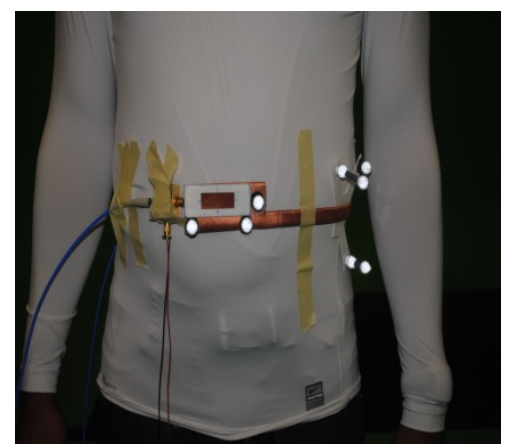

(a)

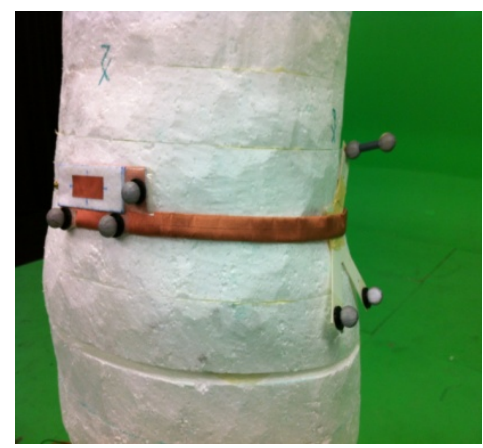

(b)

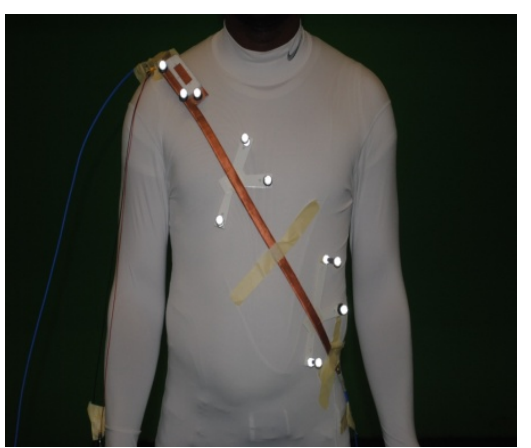

(c)

$\left|\mathrm{S}_{11}\right|$ and $\left|\mathrm{S}_{21}\right|$ were measured 10 times and the average of each was calculated. Similar measurements were taken using the polystyrene torso. The polystyrene phantom was $110 \mathrm{~cm}$ above the ground with a circumference of $90.4 \mathrm{~cm}$. The torso levels of both models were identical but their circumferences were different by $12 \%$.

\subsection{Simulation}

Once the 3D-body model was scanned and processed, the phantom data was imported into the simulation software (CST). As shown in Figure 3, the antenna was mounted on the body phantom in the same location as the measurement, and bent into the approximate shape according to the coordinate data recorded by the motion capture system. The simulated body phantom was defined as a homogenous material with a dielectric value of 35.15 and a conductivity of $1.159 \mathrm{~S} / \mathrm{m}$ (2/3 that of muscle). A multilayer body structure was also investigated to examine the accuracy of the simulation. As shown in Figure 3c, the internal muscle phantom was covered by a $12 \mathrm{~mm}$ intermediate layer of fat and a $4 \mathrm{~mm}$ external layer of dry skin. Fat has a relative permittivity of 5.28 and a conductivity of 0.105 at $2.45 \mathrm{GHz}$, and dry skin has a relative permittivity of 38 and a conductivity of 1.464 [27]. The simulated antenna return loss with the multilayer phantom was almost the same as the result from homogenous phantom. The antenna gain was slightly increased by $0.2 \mathrm{~dB}$ with the multilayer structure. 
Figure 3. (a) and (b) The scanned 3D-body model data imported into simulation software, the antenna was attached in the same location as in the measurement (c) Multi-layer numerical phantom (d) Antenna $\mathrm{X}-\mathrm{Z}$ plane radiation pattern with homogenous and multi-layer phantoms.

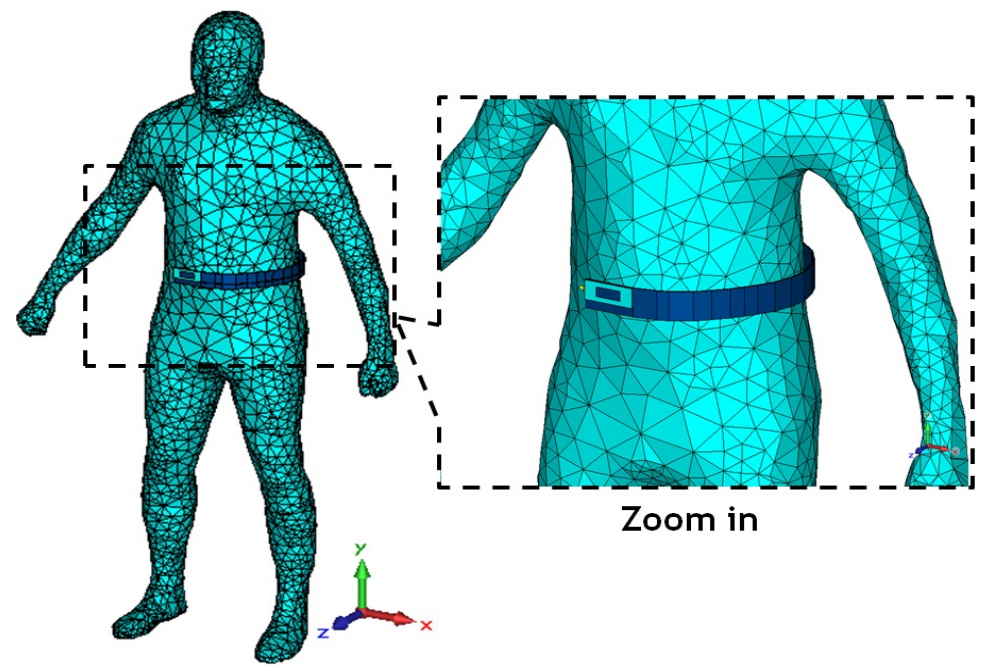

(a)

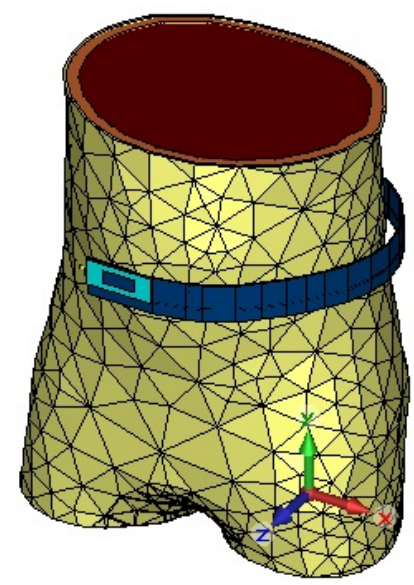

(c) (b)

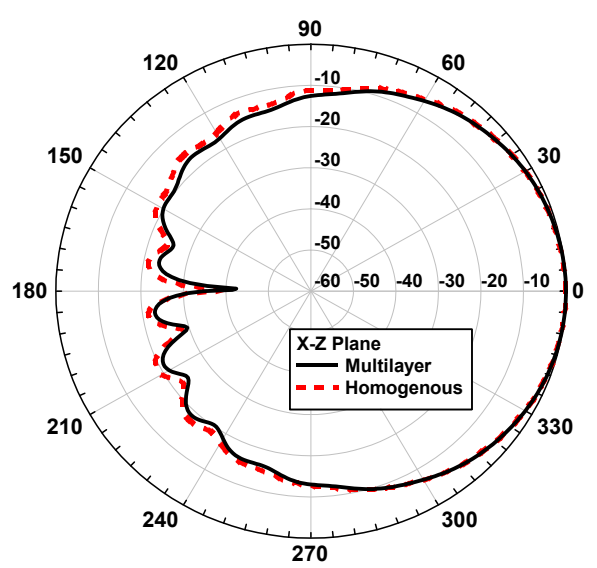

(d)

\section{Switchable Textile Antenna Design}

The switchable antenna and stripline system has overall dimensions of $45 \mathrm{~mm}$ by $600 \mathrm{~mm}$ with $4.5 \mathrm{~mm}$ thickness. This antenna consists of two parts. As shown in Figure 4a, an aperture coupled microstrip patch antenna located on the top left corner is designed for the $2.45 \mathrm{GHz}$ off-body communications and a $600 \mathrm{~mm}$ long strip line connects the port 1 and port 2 for the on-body applications. Two PIN diodes are used to switch this antenna system between on and off-body modes. PIN diode 1 controls the connection between the feed and the patch antenna, and the PIN diode 2 connects the feed to the transmission stripline. In the measurement, a power supply unit with two independent outputs is used to actuate PIN1 and PIN2 separately. The conducting fabric called pure Copper Taffeta is used in this design to replace all the metallic parts, which is a high quality Polyester based substrate plated with copper with a conductivity of $5 \mathrm{MS} / \mathrm{m}$. The dielectric part in this antenna uses the textile material Felt, which has the relative permittivity of 1.38 and a loss tangent of 0.023 . 
More details about the antenna geometry and materials can be found in our previous work [22]. Compared to wireless solutions, this stripline solution can significantly reduce the path loss, especially to overcome the high attenuations occurred in non-line-of-sight cases. Furthermore, the main part of the communication system is only $2.2 \mathrm{~mm}$ thick and made of pure flexible textile materials which indicate the antenna can be seamlessly integrated into garments without affecting the garments outside appearance or adding to the weight.

Figure 4. The antenna system and the switching network.

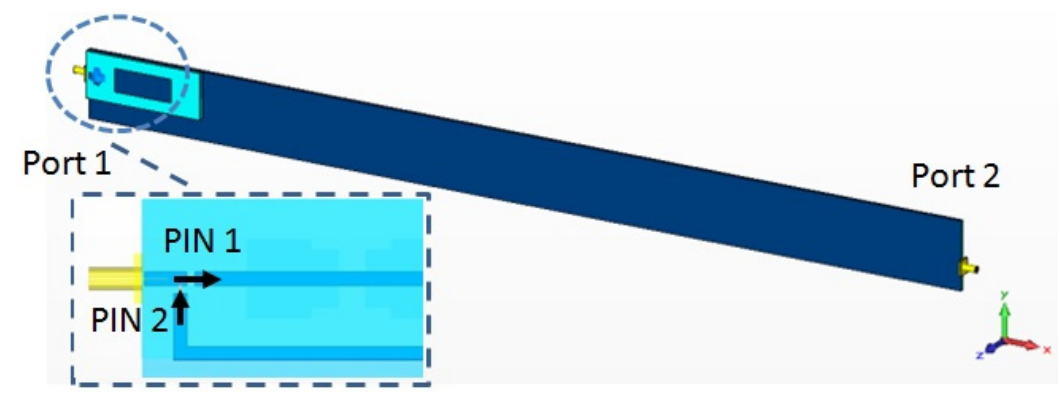

(a)

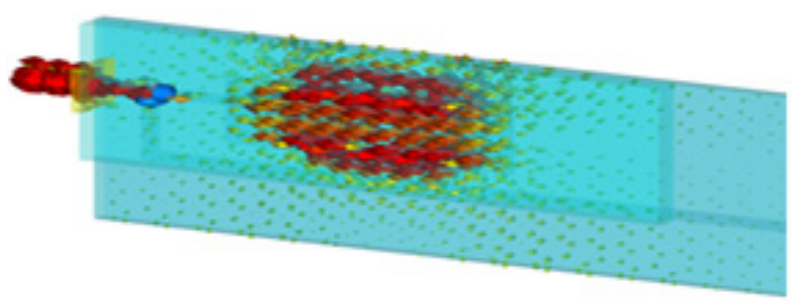

(b)

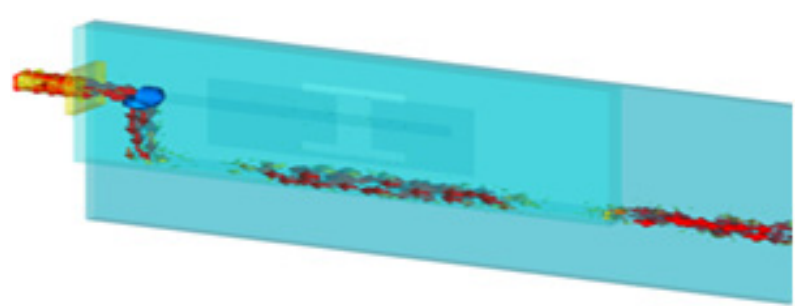

(c)

Figure $4 b, c$ present the antenna surface current distribution pattern in the antenna off-body mode and on-body mode correspondingly. When the PIN1 is on (short) and PIN2 is off (open), the antenna system is working in off-body mode, where we can see the strong surface current concentrated on the patch component (Figure $4 \mathrm{~b}$ ). The measured $\mathrm{S}$ parameter results show the antenna has a $-10 \mathrm{~dB}$ bandwidth from $2.38 \mathrm{GHz}$ to $2.51 \mathrm{GHz}$, which totally covers the $2.45 \mathrm{GHz}$ frequency ISM band $(2.4 \sim 2.5 \mathrm{GHz})$. No more than $-22 \mathrm{~dB}$ is transmitted to port 2, which indicates this antenna system radiates most of the energy through the patch antenna (Figure 5). The antenna system is operating in on-body mode when the PIN1 is off and PIN2 is on. The feeding signal is mainly transmitted to Port 2 through the stripline instead of being radiated to the air off body. The measured results also show the $\left|\mathrm{S}_{21}\right|$ is around $-5 \mathrm{~dB}$ within the frequency range 2-3 GHz while the reflection coefficient is lower than $-27 \mathrm{~dB}$. 
Figure 5. Measured reflection and transmission coefficients in antenna on-body and off-body modes.

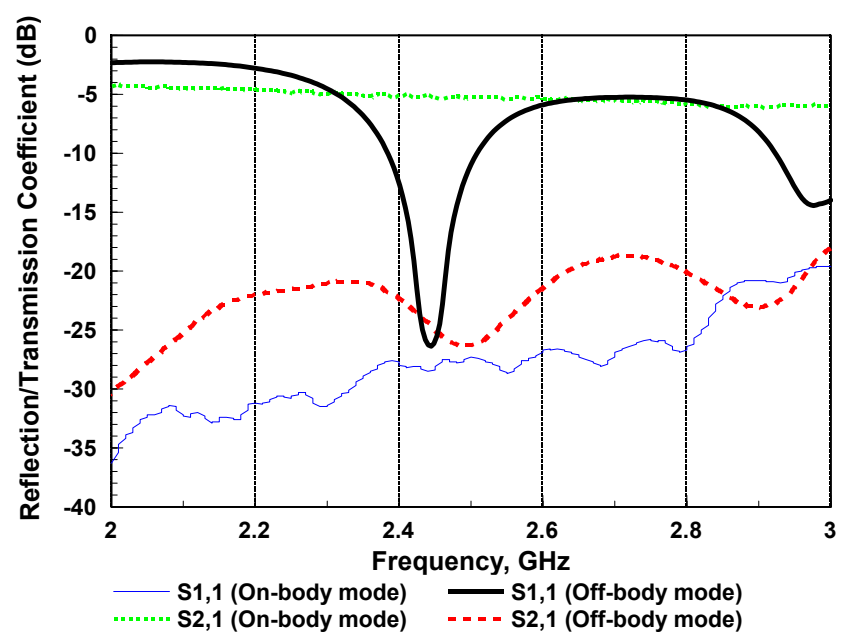

\section{System On-Body Mode}

One of the advantages of this wearable communication system is that the body effect can be neglected in the on-body mode communication. That is because in the on-body mode the signals are only transmitted through the stripline which is completely wrapped by the ground layer. However, the long and thin stripline is more likely to experience shape distortions in the realistic usage.

Figure 6. (a) stripline in completely straight condition; (b) wrapping around poly-form with $80 \mathrm{~mm}$ diameter; (c) bending around poly-form with $140 \mathrm{~mm}$ diameter; (d) twisting and bending around poly-form with $80 \mathrm{~mm}$ diameter; (e) measured transmission coefficient.
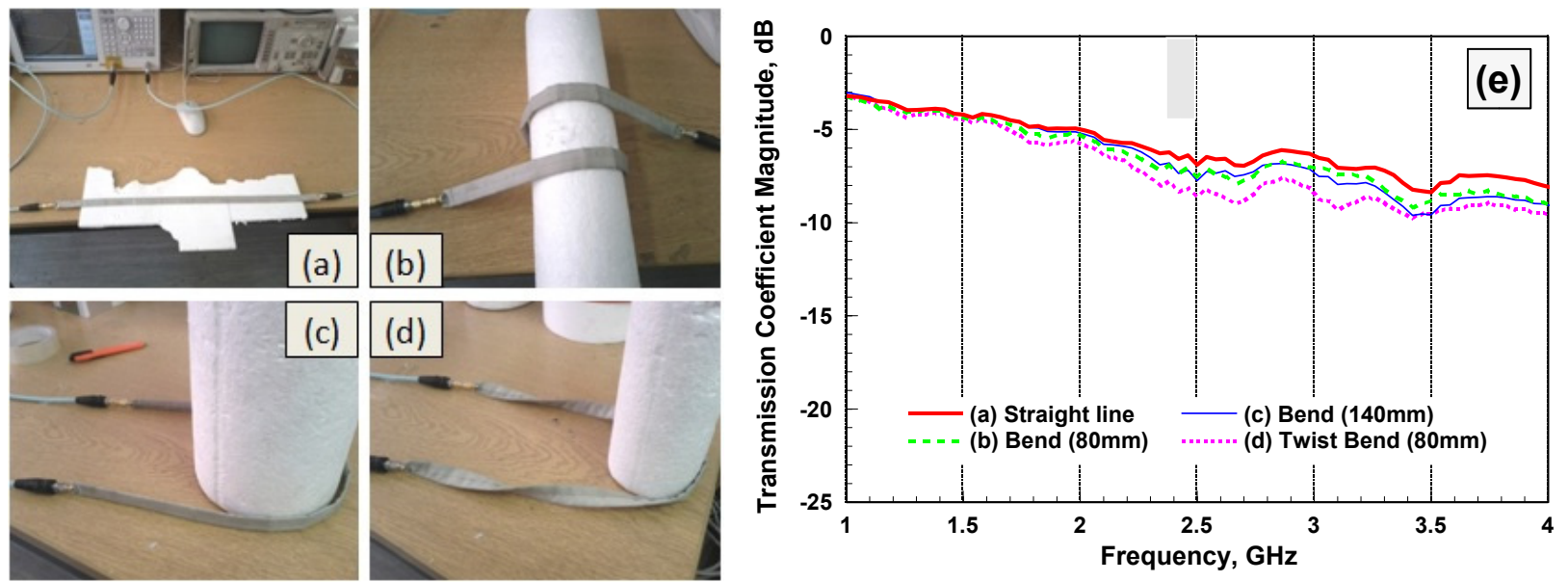

The flexible textile stripline may be curved when it is mounted on the trunk of a human body, and will be bent if it is wrapped around the waist. If the stripline is put on relatively small body components, such as wrist or joints, then some severe deformation forms, such as sharp bending and twisting, may take place during the body movement. Therefore, this section will investigate the stripline transmission performance under various shape deformation conditions. Firstly, the distortion study started from some "gentle" deformation forms. As shown in Figure 6, the $600 \mathrm{~mm}$ long stripline 
was bent around cylinders with 80 and $140 \mathrm{~mm}$ diameters (Figure 6b,c) which indicate the sizes of an adult upper arm and thigh. Comparing to the straight condition, the stripline transmission coefficient dropped by up to $0.8 \mathrm{~dB}$ within the $2.4 \mathrm{GHz}$ ISM band in both bending cases (Figure 6e). The twisting can also be combined with bending deformation as shown in Figure $6 \mathrm{~d}$, and cause $1 \mathrm{~dB}$ more attenuation in the transmission coefficient. Results show the stripline performance will only be slightly affected in the gentle bending and twisting distortion cases.

Four severe deformation forms were also investigated in this research, as shown in Figure 7: sharp bending, crumpling and squashed crumpling ( 3 and 7 bends). In the crumpling and sharp bending cases (Figure 7a,b), the stripline retains similar performance as in the straight condition with up to $1 \mathrm{~dB}$ additional loss within the $2.4 \mathrm{GHz}$ ISM band. Squashed crumpling may be considered as the most severe distortion conditions, which is very rare in the realistic usage, but can be used to evaluate the stripline performance under extreme distortion conditions. Two squashed crumpling forms were looked into: one with 3 bends and the other one has 7 bends where the $600 \mathrm{~mm}$ long stripline was almost reeled together (Figure 7c,d). Comparing to the straight condition, the 3 bends distortion will bring up to $2 \mathrm{~dB}$ additional losses to the stripline transmission. However, the stripline performance was obviously affected in the 7 bends case, where a $3 \mathrm{~dB}$ coefficient deduction was noticed in the band of interest, and the overall transmission coefficient curve was altered (Figure 7e).

Figure 7. (a) stripline sharp bending; (b) stripline crumpling; (c) squashed crumpling with 3 bends; (d) squashed crumpling with 7 bends; (e) measured transmission coefficient.
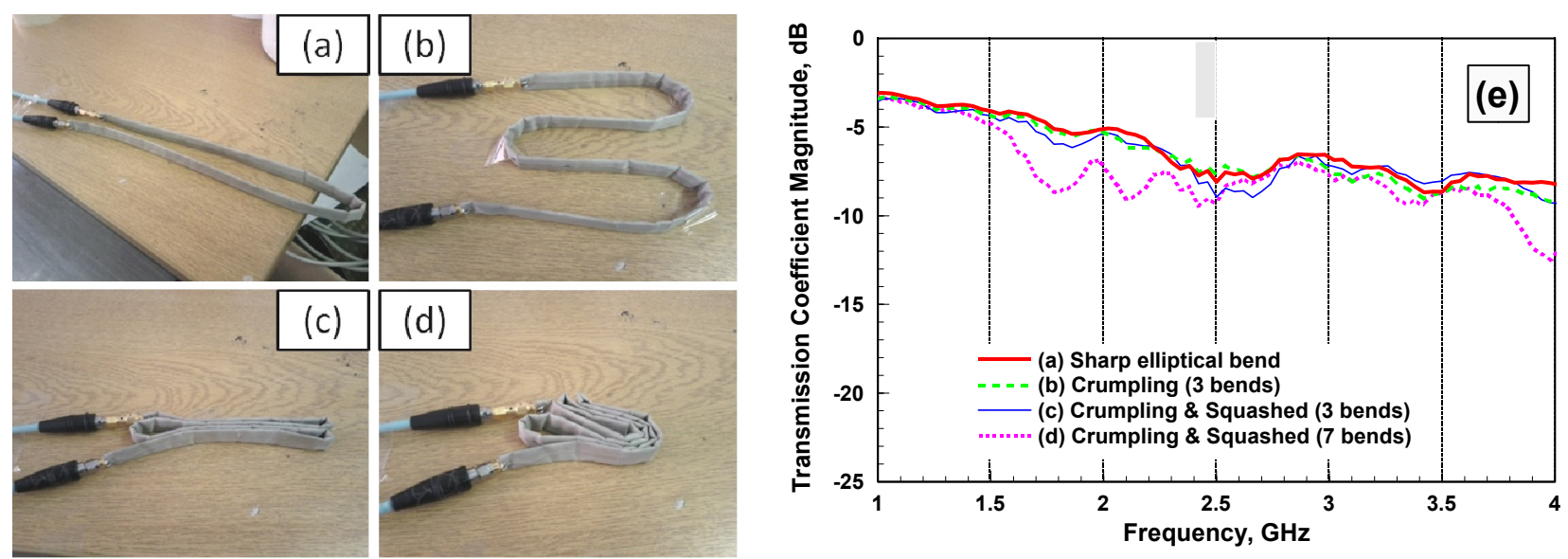

\section{System Off-Body Mode}

The communication system turns to the off-body mode when the PIN1 is on and PIN2 is off, where the system is acting as a patch antenna and communicating singles with the nodes far away from human body. In the off-body mode, the human body effects have to be taken into consideration as both the antenna matching and radiation performances can be affected by the human body. In addition, the human body surface is not in a completely flat condition, which means mounting the flexible textile antenna on human body may bend the antenna along the body curved surface. Owing to the relatively compact size of this antenna design, the patch part was only slightly bent when it was mounted on the human torso. To evaluate the antenna performance under the bending condition, a polystyrene torso model was used. The antenna system was wrapped around the torso waist with the patch antenna 
component in the center front of the torso model (Figures $2 \mathrm{~b}$ and 3 ). Figure $8 \mathrm{a}$ presents the measured $\mathrm{S}$ parameter results, from where we can see that the antenna $-10 \mathrm{~dB}$ working band covered from $2.38 \mathrm{GHz}$ to $2.50 \mathrm{GHz}$ under the flat condition, and was shifted upwards by $20 \mathrm{MHz}$ when mounted on polystyrene torso. The transmission coefficient also dropped by $1.5 \mathrm{~dB}$ within the working frequency band due to the stripline being bent around the torso. The simulation presents a very good agreement with the measurement results, where the $3 \mathrm{D}$ laser scanner and the motion capture system played an important role to minimize the difference between measurement and simulation.

Figure 8. Antenna reflection and transmission coefficients in flat condition and on polystyrene torso: (a) Measurement; (b) simulation.

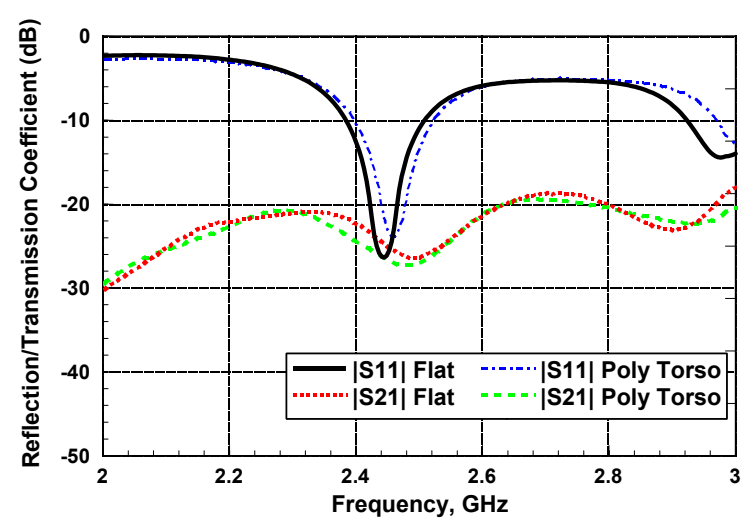

(a)

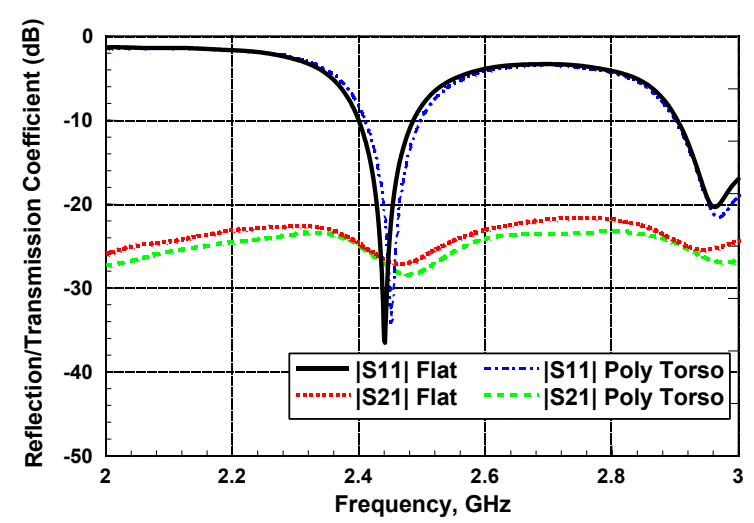

(b)

Then the antenna was tested on a real human body, and an additional antenna position, diagonally crossing the human chest with the patch antenna component on the right shoulder (Figure 2c), was investigated. From Figure 9, it can be seen that placing the antenna on the real human subject or on the polystyrene model caused the reflection and transmission coefficients to change only slightly over the 2-3 GHz frequency band of interest (Figure 9a). Results show that $12 \mathrm{~cm}$ difference in torso circumference and the difference between human tissues properties and polystyrene properties only cause $4 \mathrm{MHz}$ broadening on the $-10 \mathrm{~dB}$ working bandwidth in the "bending around waist" case. When the patch antenna was put on the shoulder with stripline diagonally crossing the body chest, the bandwidth was also widened by $4 \mathrm{MHz}$ and the resonant frequency was shifted upwards by $13 \mathrm{MHz}$. It can be concluded that the matching performance of this antenna system is fairly robust for body-worn applications.

The wearable antenna radiation pattern may be affected by the human body as the body can be considered as a large dielectric material with a high relative permittivity [2,7]. Furthermore, the wearer may keep changing posture during daily activities, which will create a dynamic operating environment for the wearable antenna. In other words, the wearable antenna, even in a fixed on-body location, can experience varying body effects during the wearer's movement. 
Figure 9. Antenna reflection and transmission coefficients on real human and polystyrene model: (a) bending around waist; (b) diagonally crossing the body chest.

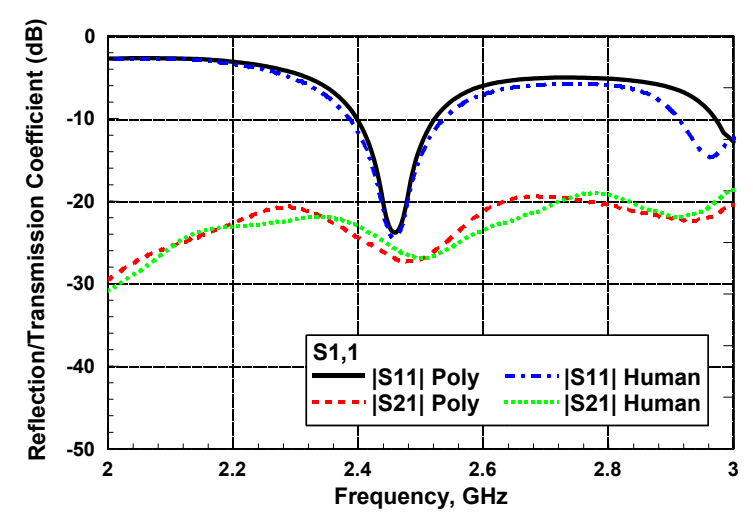

(a)

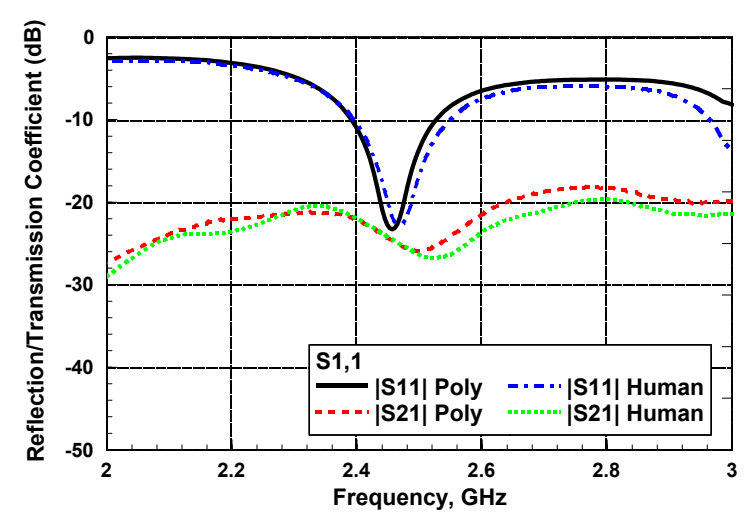

(b)

Figure 10. Simulated and measured radiation patterns of antenna, azimuth plane, normalized to the maximum value in: (a) Simulated in free space and on human body; (b) On stomach bending forward upper part of body (measurement); (c) On right shoulder tilting head (measurement); (d) On right shoulder turning head (measurement).

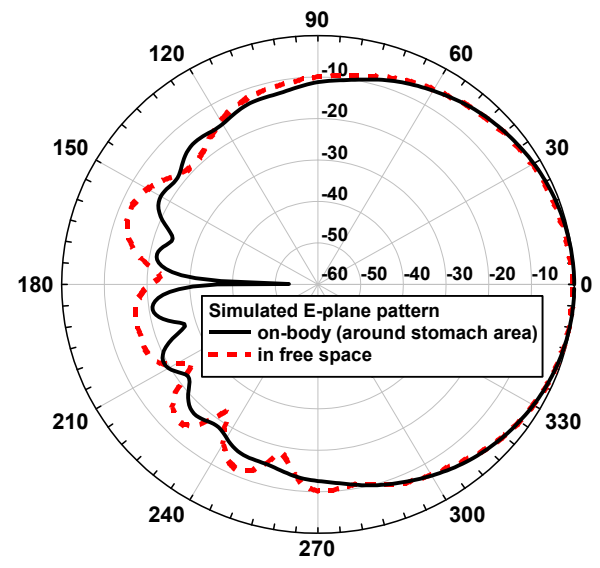

(a)

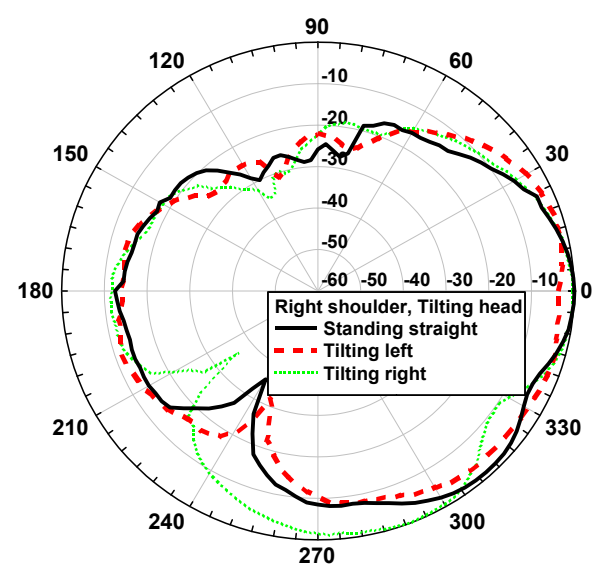

(c)

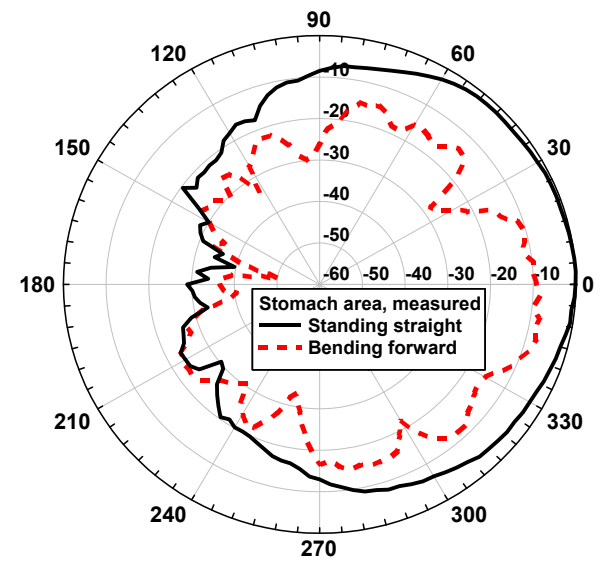

(b)

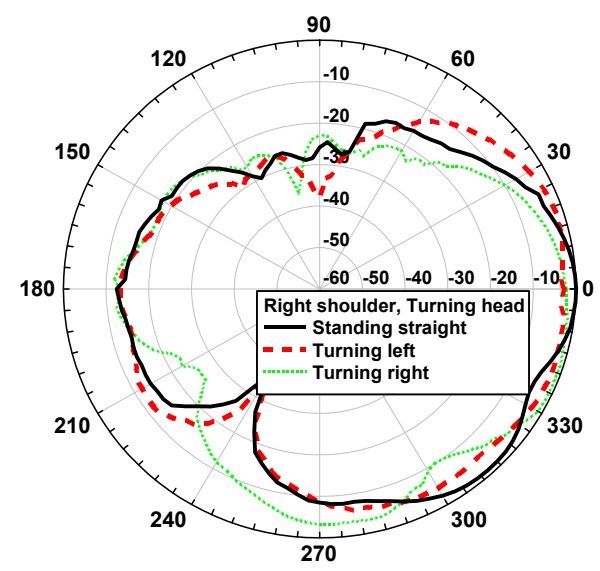

(d) 
Figure 10a presents the simulated antenna E-plane radiation patterns of the antenna in free space and on the human body at $2.45 \mathrm{GHz}$. The antenna absolute gain is $1.48 \mathrm{dBi}$ in free space. Comparing to the pattern in free space, we can see the antenna forward gain was reduced by $0.6 \mathrm{~dB}$ when it was attached to the human body, while there was a $6 \mathrm{~dB}$ reduction on the backward radiation. The antenna radiation efficiency also dropped from $43 \%$ to $39 \%$ when the antenna was in the proximity of the human body. To evaluate the maximum effects which the body movement may bring to the radiation performance, the antenna was tested not only with a standing still human subject, but also with some typical body movements, for example bending forward the upper part of body by $80^{\circ}$ or tilting/turning the head. Figure $10 \mathrm{~b}-\mathrm{d}$ present the measured radiation pattern when the antenna is put in the center front of the stomach area (Figure 2a) and on the right shoulder (Figure 2c). Only the azimuth plane is measured due to the limitation of the measurement equipment. It is noticed that the head movements only caused slight changes in the radiation pattern when the antenna was put on the right shoulder (Figure 10c,d). The simulation results show the antenna radiation efficiency has only a $3 \%$ variation for the head movement cases. However, a severe body effect was found when bending forward the upper part of body, which could cause a $10 \mathrm{~dB}$ reduction to the antenna forward gain when the patch antenna was attached in the center front of the human stomach (Figure 10b).

\section{Conclusions}

A switchable textile antenna and stripline communication system operating at $2.45 \mathrm{GHz}$ was presented at different locations on the body with detailed investigation of a realistic on-body environment in different postures. In the on-body mode, the system transmission performance was only slightly affected by the human body, and remained stable under shape distortion conditions. In the off-body mode, the patch antenna resonant frequency was shifted upwards by $13 \mathrm{MHz}$ when mounted on a human body, but still fully covered the $2.45 \mathrm{GHz}$ ISM band. The antenna radiation performance was varying with the movement of wearer, where bending the upper part of the body forward could give up to $10 \mathrm{~dB}$ gain reductions if the patch antenna was attached around the stomach area. Numerical and experimental results show an excellent agreement, and indicate that it is possible to employ textile materials to realize a robust communication performance for wearable applications.

\section{Acknowledgments}

This work is funded by the UK Engineering and Physical Sciences Research Council (EPSRC), grant reference number: EP/G056633.

\section{Author Contributions}

HJL and QB were responsible for the system design, simulation and free space measurement, which were supervised by KLF and RJL in University of Sheffield. The human body scan and on-body measurement were carried out by SS, supervised by JCB, in University of Kent. All authors contributed equally to data analysis, interpretation of results and manuscript preparation. 


\section{Conflicts of Interest}

The authors declare no conflict of interest.

\section{References}

1. Hall, P.S.; Hao, Y. Antennas and Propagation for Body-Centric Wireless Networks, 2nd ed.; Artech House: Norwood, MA, USA, 2012.

2. Hall, P.S. Antennas challenges for body centric communications. In International Workshop on Antenna Technology (IWAT'07), Cambridge, UK, 21-23 March 2007; pp. 41-44.

3. Bai, Q.; Langley, R.J. Crumpling of PIFA textile antenna. IEEE Trans. Antenna Propag. 2012, 60, 63-70.

4. Salonen, P.; Jasehoon, K.; Rahmat-Sammi, Y. Dual-band E-shaped patch wearable textile antenna. In Proceedings of the Antennas and Propagation Society International Symposium, Washington, DC, USA, 3-8 July 2005; Volume 1A, pp. 466-469.

5. Zhu, S.; Langley, R. Dual-band wearable antennas over EBG substrate. IET Electron. Lett. 2007, 43,3 .

6. Boeykens, F.; Rogier, H.; Vallozzi, L. An efficient technique based on polynomial chaos to model the uncertainty in the resonance frequency of textile antennas due to bending. IEEE Trans. Antennas Propag. 2014, 62, 1253-1260.

7. Hertleer, C.; Rogier, H.; Langenhove, L.V. The effect of moisture on the performance of textile antennas. In Proceedings of the 2nd IET Seminar on Antennas Propagation for Body-Centric Wireless Communications, Stevenage, UK, 20 April 2009; p. 1.

8. Lilja, J.; Salonen, P.; Kaija, T.; de Maagt, P. Design and manufacturing of robust textile antennas for harsh environments. IEEE Trans. Antennas Propag. 2012, 60, 4130-4140.

9. Klemm, M.; Locher, I.; Troster, G. A novel circularly polarized textile antenna for wearable applications. In Proceedings of the 34th European Microwave Conference, Amsterdam, The Netherlands, 12-14 October 2004; Volume 1, pp. 137-140.

10. Chandran, A.R.; Scanlon, W.G. Dual-band low profile antennas for body-centric communications. In International Workshop on Antenna Technology (IWAT'10), Lisbon, Portugal, 1-3 March 2010; pp. 1-4.

11. Kennedy, T.F.; Fink, P.W.; Chu, A.W.; Champagne, N.J.; Lin, G.Y.; Khayat, M.A. Body-worn E-textile antennas: The good, the low-mass, and the conformal. IEEE Trans. Antennas Propag. 2009, 57, 910-918.

12. Abbasi, Q.H.; Rehman, M.U.; Yang, X.; Alomainy, A.; Qaraqe, K.; Serpedin, E. Ultrawideband band-notched flexible antenna for wearable applications. IEEE Antennas Wirel. Propag. Lett. 2013, 12, 1606-1609.

13. Khouri, R.; Ratajczak, P.; Brachat, P.; Staraj, R. A thin surface-wave antenna using a via-less EBG structure for $2.45 \mathrm{Ghz}$ on-body communication systems. In Proceedings of the 4th European Conference on Antennas and Propagation (EuCAP), Barcelona, Spain, 12-16 April 2010; pp. 1L-4L. 
14. Akhoondzadeh-Asl, L.; Hall, P.S.; Nechayev, Y. Novel conformal surface wave Yagi antenna for on-body communication channel. In Proceedings of the IEEE International Symposium on Antennas and Propagation (APSURSI), Toronto, ON, Canada, 11-17 July 2010; pp. 1-4.

15. Lin, C.-H.; Saito, K.; Takahashi, M.; Ito, K. A compact planar Inverted-F antenna for $2.45 \mathrm{GHz}$ on-body communications. IEEE Trans. Antennas and Propag. 2012, 60, 4422-4426.

16. Chahat, N.; Zhadobov, M.; le Coq, L.; Sauleau, R. Wearable endfire textile antenna for on-body communications at $60 \mathrm{GHz}$. IEEE Antennas Wireless Propag. Lett. 2012, 11, 799-802.

17. Conway, G.A.; Scanlon, W.G. Antennas for over-body-surface communication at $2.45 \mathrm{GHz}$. IEEE Trans. Antennas Propag. 2009, 57, 4.

18. Chandran, A.R.; Conway, G.A.; Scanlon, W.G. Pattern switching compact patch antenna for on-body and off-body communications at $2.45 \mathrm{GHz}$. In Proceedings of the 3rd European Conference on Antennas and Propagation (EuCAP), Berlin, Germany, 23-27 March 2009; pp. 2055-2057.

19. Kelly, J.; Ford, K.L.; Langley, R.J. Slotline structure for on/off-body communications at $2.45 \mathrm{GHz}$. In Proceedings of the 5th European Conference on Antennas and Propagation (EUCAP), Rome, Italy, 11-15 April 2011; pp. 525-529.

20. Langley, R.J.; Ford, K.L.; Lee, H.-J. Switchable on/off-body communication at $2.45 \mathrm{GHz}$ using textile strip patch antenna on stripline. In Proceedings of the 2012 6th European Conference on Antennas and Propagation (EUCAP), Prague, Czech Republic, 26-30 March 2012; pp. 728-731.

21. Bai, Q.; Ford, K.L.; Langley, R.J. Switchable surface wave antenna for On/off-body communications. In Proceedings of the European Conference on Antennas \& Propagation (EUCAP), Gothenburg, Sweden, 8-12 April 2013; pp. 1644-1647.

22. Lee, H.J.; Ford, K.L.; Langley, R.J. Switchable on/off-body communication at $2.45 \mathrm{GHz}$ using textile strip patch antenna on stripline. Electron. Lett. 2012, 48, 254-256.

23. Human Solutions. Body Scanner. Available online: http://www.human-solutions.com/fashion/ front_content.php?idcat=126 (accessed on 16 January 2013).

24. Lerch, T.; MacGillivrary, M.; Domina, T. 3D laser scanning: A model of multidisciplinary research. J. Text. Appar. Technol. Manag. North Carol. State Univ. 2007, 5, 1-22.

25. Swaisaenyakorn, S.; Young, P.R.; Kelly, S.W.; Batchelor, J.C. Evaluation of 3D animated human model from 3D scanner and motion capture to be used in electromagnetic simulator for body-centric system. In Proceedings of the 5th International Conference on BioMedical Engineering and Informatics, Chongqing, China, 16-18 October 2012.

26. Swaisaenyakorn, S.; Batchelor, J.C. Body gain study for animated human model in a body worn antenna system. In Proceedings of the 2012 IEEE Asia-Pacific Conference on Antennas and Propagation (APCAP), Singapore, Singapore, 27-29 August 2012; pp. 108-109.

27. Italian National Research Council, Institute for Applied Physics. Available online: http://www.ifac.cnr.it/ (accessed on 1 June 2014).

(C) 2014 by the authors; licensee MDPI, Basel, Switzerland. This article is an open access article distributed under the terms and conditions of the Creative Commons Attribution license (http://creativecommons.org/licenses/by/3.0/). 